\title{
Estudo cefalométrico das alturas faciais anterior e posterior, em crianças brasileiras, portadoras de má oclusão Classe I de Angle, na fase de dentadura mista
}

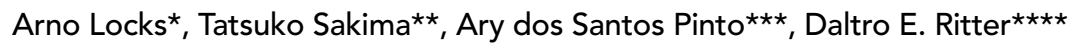

\begin{abstract}
Resumo
Realizou-se um estudo das alturas faciais anterior e posterior em 79 crianças brasileiras, sendo 46 do gênero feminino e 33 do masculino, com idades de 8 a 11 anos, portadoras de má oclusão Classe I de Angle, na fase de dentadura mista, não submetidas a tratamento ortodôntico. Esta investigação utilizou duas radiografias cefalométricas em norma lateral, de cada criança, com intervalo de 8 a 16 meses entre uma radiografia e outra, para comparação dos dados cefalométricos. Foram estudadas as proporções faciais anteriores, ou seja, a relação entre as dimensões N-Me (AFT), N-ENA (AFS), ENA-Me (AFI) e o relacionamento entre as dimensões Ar-GO (AFP) e a distância entre o plano palatino ao mento (AFA) , isto é, o Índice da Altura Facial (IAF). Os resultados obtidos, possibilitaram constatar que as tendências de crescimento foram equilibradas em todas as situações estudadas; a dimensão altura facial inferior, contribuiu de forma mais significativa, nas alterações observadas na altura facial total; as proporções faciais anteriores se mantiveram em torno de $42 \%$ para a altura facial superior e $58 \%$ para a altura facial inferior; o índice da altura facial ou se manteve constante em torno de 0,66 ou apresentou uma tendência a aumentar com a idade; não ocorreu dimorfismo sexual com relação às proporções faciais e índice da altura facial.
\end{abstract}

Palavras-chave: Cefalometria. Crescimento. Padrão esquelético

\section{INTRODUÇÃO E REVISÃO DE LITERATURA}

São muitas as pesquisas realizadas até os dias de hoje, buscando aprimorar os nossos conhecimentos científicos relacionados à Cefalometria. Como a mais popular classificação de má oclusão, idealizada por Angle, foi definida somente na dimensão ântero-posterior, é natural que a atenção dos investigadores estivesse voltada, principalmente, para o equilíbrio ântero-posterior do complexo dentofacial ${ }^{27}$. No entanto, a maioria das más oclusões apresenta alterações tanto no sentido vertical quanto no sentido

* Doutor pela UNESP - Araraquara-SP. Pós Doutorado na Universidade de Aarhus, Dinamarca. Professor Adjunto da Disciplina de Ortodontia da UFSC-SC. Coordenador do Curso de Especialização em Ortodontia da UFSC-SC. Mestre pela UFRJ - RJ.

** Professor Titular da Disciplina de Ortodontia da UNESP - Araraquara - SP.

*** Professor Adjunto da Disciplina de Ortodontia da UNESP - Araraquara - SP.

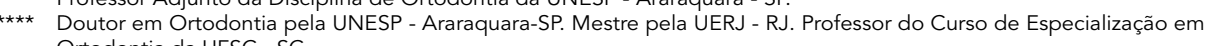
Ortodontia da UFSC - SC. 
ântero posterior ${ }^{2}$. O estudo do equilíbrio vertical da face é extremamente útil no diagnóstico e no plano de tratamento, sendo que as más oclusões com acentuado desequilíbrio vertical são, geralmente, mais difíceis de se tratar do que aquelas com discrepância ântero posterior ${ }^{23}$. Há um padrão facial médio para haver oclusão excelente ${ }^{8}$ e o padrão normal é equilíbrio e harmonia de proporções ${ }^{34}$. O conhecimento do crescimento facial, com a manutenção ou não das proporções faciais é de suma importância para o ortodontista.

Os indivíduos variam no tipo e padrão faciais, mas aqueles que apresentam equilíbrio e estética facial tem certas características comuns de perfil ${ }^{9}$. A aparência física é de extrema importância na sociedade $^{36}$, e portanto o ortodontista deve entender a beleza facial assim como ela é concebida pelo olhar do público em geral, procurando o equilíbrio, harmonia e proporção facial.

Quando acompanhamos o crescimento normal de um indivíduo, evidenciamos que o padrão de desenvolvimento facial segue um curso regular e ordenado, desde a infância até a fase adulta ${ }^{03,04}$, apesar de haver alterações na velocidade de crescimento de acordo com a idade ${ }^{38}$. O padrão de desenvolvimento de cada forma facial, é estabelecido em um idade bastante precoce ${ }^{24}$, e mantêm proporções mais ou menos equilibradas de acordo com a idade $30,32,38,37,03,33,07,24,04,15$.

Com relação à altura facial total anterior (N-Me), os indivíduos do gênero masculino, apresentam esta dimensão, geralmente maior do que os do gênero feminino ${ }^{6,17,20,05,22,38,23,28}$.

Quando ocorrem alterações nas proporções faciais anteriores, a dimensão altura facial inferior (ENA-Me) é a que contribui mais com estas alterações ${ }^{13,19,14,31,26,29,25,10,30,18,21}$, permanecendo a altura facial superior (N-ENA) com poucas modificações.

A altura facial posterior (Ar-Go) aumenta mais do que a altura facial anterior (distância do plano palatino até o ponto mento), nos casos com crescimento harmonioso, ocasionando uma rotação da mandíbula no sentido anti-horário ${ }^{12,35}$.

$O$ uso do índice da altura facial (IAF), (razão entre AFP e AFA) é bastante útil durante o tratamento da má oclusão Classe II, com ângulo FMA alto ${ }^{16}$. Se a AFP aumenta mais rapidamente do que a AFA, durante o tratamento ortodôntico e/ou crescimento, o padrão facial do paciente com a má oclusão Classe II melhora, porque a rotação da mandíbula ocorreu para cima e para frente. Ao contrário, se a AFA aumentar mais do que a AFP, a mandíbula terá rotação para baixo e para trás. Portanto, durante o tratamento ortodôntico, o objetivo seria manter a AFA e aumentar a AFP. Este índice pode ajudar o clínico no plano de tratamento, cujos casos poderão ser somente ortodônticos, (índice entre 0,55 e 0,85) ou cirúrgicos, quando o índice está fora destes limites de variação ${ }^{16}$.

A finalidade deste trabalho, foi realizar um estudo cefalométrico das alturas faciais, anterior e posterior, em indivíduos na fase de dentadura mista, e não submetidos a tratamento ortodôntico.

\section{MATERIAIS E MÉTODOS}

O material empregado na realização deste trabalho, constou de 158 telerradiografias cefalométricas em norma lateral, obtidas de 79 crianças brasileiras, leucodermas, com idades entre 8 e 11 anos, sendo 33 do gênero masculino e 46 do gênero feminino. Todas as crianças eram portadoras de má oclusão Classe I de Angle, e não haviam sido submetidas a tratamento ortodôntico. Elas foram selecionadas nos arquivos de documentação da Disciplina de Ortodontia Preventiva, do Departamento de Clínica Infantil da Faculdade de Odontologia de Araraquara, dentre aquelas que se inseriam nos parâmetros previamente estipulados. De cada criança selecionada obtiveram-se duas radiografias cefalométricas com um intervalo de no mínimo 8 meses e no máximo 16 meses. Todas as estruturas anatômicas e os pontos cefalométricos foram identificados três vezes, obedecendo a um intervalo de um mês, 
entre cada vez, com a finalidade de minimizar os possíveis erros de identificação.

A amostra foi dividida em 4 períodos, sendo que cada período era constituído de um instante inicial e um final mantendo-se um intervalo de 8 a 16 meses entre o instante inicial e o final, para cada período, da seguinte maneira:

\section{Período I}

Instante Inicial (Ii) - crianças com idade de 8 a 8 anos e 6 meses.

Instante Final (If) - mesmas crianças com idade de 9 a 9 anos e 6 meses.

\section{Período II}

Instante Inicial (IIi) - crianças com idade de 8 anos e 6 meses a 9 anos.

Instante Final (IIf) - mesmas crianças com idade de 9 anos e 6 meses a 10 anos.

\section{Período III}

Instante Inicial (IIIi) - crianças com idade de 9 a 9 anos e 6 meses.

Instante Final (IIIf) - mesmas crianças com idade de 10 a 10 anos e 6 meses.

\section{Período IV}

Instante Inicial (IVi) - crianças com idade de 9 anos e 6 meses a 10 anos.

Instante Final (IVf) - mesmas crianças com idade de 10 anos e 6 meses a 11 anos.

\section{PLANEJAMENTO ESTATíSTICO}

Empregou-se modelo de regressão linear, com intervalo de confiança a $95 \%$ e o teste de igualdade de percentuais com a estatística $Z$ normal.

\section{RESULTADOS}

As tabelas 1, 2 e 3 mostram que houve igualdade estatística entre as médias das dimensões AFP, AFA, IAF, em todas as situações estudadas.

As médias das dimensões AFS, AFI e AFT

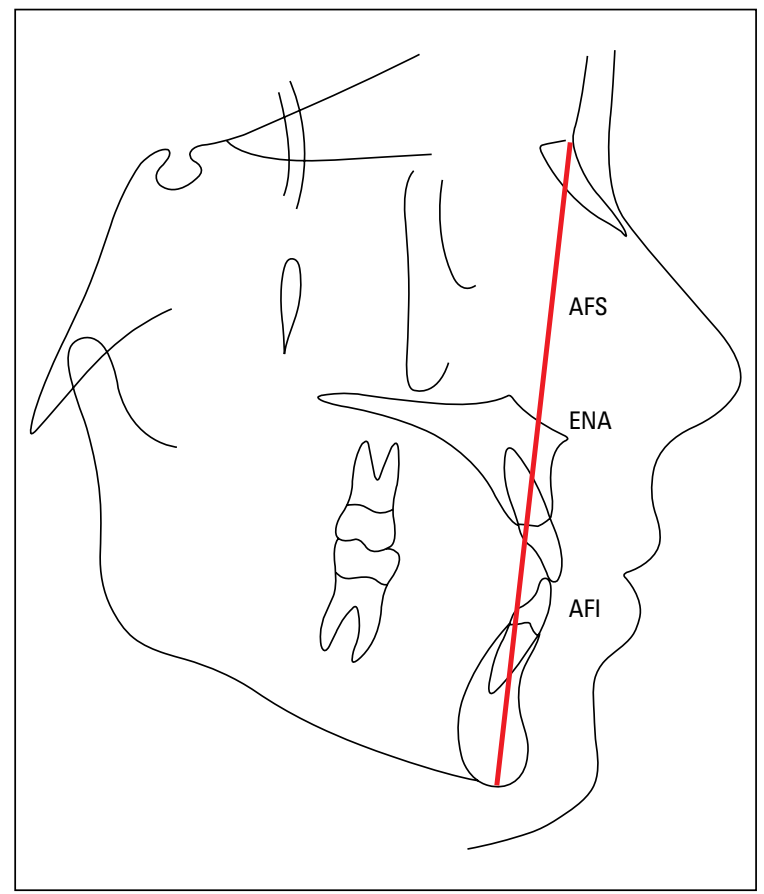

FIGURA 1 - Medidas utilizadas por Wylie e Johnson ${ }^{38}$ : AFT (distância entre os pontos N-Me), AFS (distância entre os pontos N-ENA), AFI (distância entre os pontos ENA-Me).

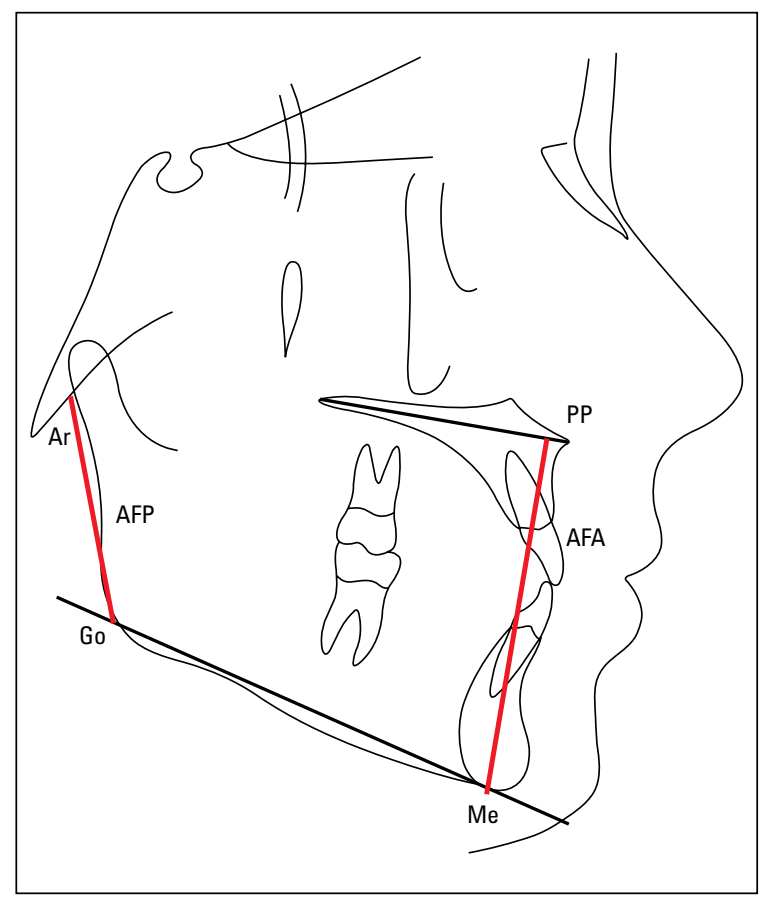

FIGURA 2 - Medidas utilizadas por Gebeck e Merrifield"11 e Horn ${ }^{16}$ : AFP (distância entre os pontos Ar-Go), AFA (distância entre o plano palatino-PP e o ponto $\mathrm{Me})$, IAF (razão entre AFP e AFA). 


\begin{tabular}{|c|c|c|c|c|c|c|c|c|c|c|}
\hline \multirow{2}{*}{ Per. } & \multirow{2}{*}{ Gênero } & \multirow{2}{*}{$\mathbf{N}$} & \multicolumn{4}{|c|}{ Instante inicial } & \multicolumn{4}{|c|}{ Instante final } \\
\hline & & & Média & E.P. & $\mathbf{L i}$ & Ls & Média & E.P. & Li & Ls \\
\hline \multirow{2}{*}{ Iff/li } & M & 11 & 38,29 & 1,10 & 35,80 & 40,78 & 39,11 & 1,06 & 36,71 & 41,51 \\
\hline & $F$ & 11 & 37,72 & 1,16 & 35,10 & 40,34 & 38,37 & 1,13 & 35,72 & 41,02 \\
\hline \multirow{2}{*}{ IIf/lli } & $M$ & 7 & 39,86 & 1,14 & 36,92 & 39,86 & 41,28 & 1,64 & 37,07 & 45,49 \\
\hline & $\mathrm{F}$ & 14 & 37,89 & 0,80 & 36,15 & 39,63 & 38,55 & 0,61 & 37,22 & 39,88 \\
\hline \multirow{2}{*}{ IIIf/IIIi } & M & 8 & 41,49 & 0,43 & 40,44 & 42,54 & 42,30 & 0,65 & 40,71 & 43,89 \\
\hline & $F$ & 10 & 40,60 & 1,65 & 36,79 & 44,41 & 41,68 & 1,53 & 38,17 & 45,19 \\
\hline \multirow{2}{*}{ IVf/IVi } & $\mathrm{M}$ & 7 & 40,54 & 0,42 & 39,46 & 41,62 & 41,36 & 0,67 & 39,64 & 43,08 \\
\hline & $\mathrm{F}$ & 11 & 39,20 & 1,08 & 36,76 & 41,64 & 39,84 & 1,05 & 37,47 & 42,21 \\
\hline \multirow{3}{*}{ (If a IVf)/(li a IVi) } & M & 33 & 39,87 & 0,49 & 38,87 & 40,87 & 40,82 & 0,56 & 39,68 & 41,96 \\
\hline & $\mathrm{F}$ & 46 & 38,75 & 0,58 & 37,55 & 39,89 & 39,50 & 0,54 & 38,41 & 40,59 \\
\hline & $\mathrm{M}+\mathrm{F}$ & 79 & 39,22 & 0,40 & 38,42 & 40,02 & 40,05 & 0,40 & 39,25 & 40,85 \\
\hline
\end{tabular}

\begin{tabular}{|c|c|c|c|c|c|c|c|c|c|c|}
\hline \multirow{2}{*}{ Per. } & \multirow{2}{*}{ Gênero } & \multirow{2}{*}{$\mathbf{N}$} & \multicolumn{4}{|c|}{ Instante inicial } & \multicolumn{4}{|c|}{ Instante final } \\
\hline & & & Média & E.P. & $L_{i}$ & $L_{s}$ & Média & E.P. & $L_{i}$ & $\mathrm{~L}_{\mathrm{s}}$ \\
\hline \multirow{2}{*}{ If/li } & M & 11 & 59,10 & 1,38 & 55,96 & 62,24 & 60,01 & 1,53 & 56,55 & 63,47 \\
\hline & $\mathrm{F}$ & 11 & 56,93 & 1,13 & 54,37 & 59,49 & 58,41 & 1,07 & 55,99 & 64,92 \\
\hline \multirow{2}{*}{ IIf/lli } & M & 7 & 60,38 & 1,47 & 56,58 & 64,14 & 61,51 & 1,59 & 57,42 & 65,60 \\
\hline & $\mathrm{F}$ & 14 & 59,39 & 1,16 & 59,86 & 61,92 & 60,45 & 1,05 & 58,16 & 62,74 \\
\hline \multirow{2}{*}{ IIIf/IIli } & $\mathrm{M}$ & 8 & 58,75 & 1,13 & 55,98 & 61,52 & 60,52 & 0,83 & 58,49 & 62,55 \\
\hline & $\mathrm{F}$ & 10 & 59,20 & 0,90 & 57,12 & 61,28 & 60,85 & 0,96 & 58,63 & 63,07 \\
\hline \multirow{2}{*}{ IVf/IVi } & M & 7 & 63,03 & 1,30 & 59,69 & 66,37 & 63,48 & 1,32 & 60,09 & 66,87 \\
\hline & $\mathrm{F}$ & 11 & 60,11 & 0,69 & 58,55 & 61,67 & 61,48 & 0,82 & 59,63 & 63,33 \\
\hline \multirow{3}{*}{ (If a IVf)/(li a IVi) } & $\mathrm{M}$ & 33 & 60,12 & 0,71 & 58,67 & 61,57 & 61,19 & 0,71 & 59,74 & 62,64 \\
\hline & $\mathrm{F}$ & 46 & 58,93 & 0,52 & 57,86 & 60,00 & 60,29 & 0,51 & 59,26 & 61,32 \\
\hline & $\mathrm{M}+\mathrm{F}$ & 79 & 59,42 & 0,43 & 58,57 & 60,29 & 60,67 & 0,42 & 59,83 & 61,51 \\
\hline
\end{tabular}

\begin{tabular}{|c|c|c|c|c|c|c|c|c|c|c|}
\hline \multirow{2}{*}{ Per. } & \multirow{2}{*}{ Gênero } & \multirow{2}{*}{$\mathbf{N}$} & \multicolumn{4}{|c|}{ Instante inicial } & \multicolumn{4}{|c|}{ Instante final } \\
\hline & & & Média & E.P. & $L_{\mathbf{i}}$ & $\mathbf{L}_{\mathrm{s}}$ & Média & E.P. & $\mathbf{L}_{\mathbf{i}}$ & $L_{s}$ \\
\hline \multirow{2}{*}{ If $/ \mathrm{li}$} & $M$ & 11 & 0,64 & 0,02 & 0,59 & 0,69 & 0,65 & 0,02 & 0,60 & 0,70 \\
\hline & $\mathrm{F}$ & 11 & 0,66 & 0,02 & 0,61 & 0,71 & 0,65 & 0,02 & 0,60 & 0,70 \\
\hline \multirow{2}{*}{ IIf/lli } & M & 7 & 0,65 & 0,02 & 0,60 & 0,70 & 0,67 & 0,03 & 0,59 & 0,75 \\
\hline & $\mathrm{F}$ & 14 & 0,63 & 0,02 & 0,59 & 0,67 & 0,63 & 0,01 & 0,61 & 0,65 \\
\hline \multirow{2}{*}{ IIIf/IIli } & $M$ & 8 & 0,70 & 0,02 & 0,65 & 0,75 & 0,70 & 0,02 & 0,65 & 0,75 \\
\hline & $\mathrm{F}$ & 10 & 0,68 & 0,02 & 0,63 & 0,73 & 0,68 & 0,02 & 0,63 & 0,73 \\
\hline \multirow{2}{*}{ IVf/IVi } & M & 7 & 0,64 & 0,01 & 0,61 & 0,67 & 0,65 & 0,01 & 0,62 & 0,68 \\
\hline & $\mathrm{F}$ & 11 & 0,65 & 0,02 & 0,60 & 0,70 & 0,64 & 0,02 & 0,59 & 0,69 \\
\hline \multirow{3}{*}{ (If a IVf) / (li a IVi) } & M & 33 & 0,66 & 0,01 & 0,64 & 0,68 & 0,66 & 0,01 & 0,64 & 0,68 \\
\hline & $\mathrm{F}$ & 46 & 0,65 & 0,01 & 0,63 & 0,67 & 0,65 & 0,01 & 0,63 & 0,67 \\
\hline & $M+F$ & 79 & 0,66 & 0,01 & 0,64 & 0,68 & 0,66 & 0,01 & 0,64 & 0,68 \\
\hline
\end{tabular}


Tabela 4 - Médias, erro-padrão e limites do intervalo segundo período, gênero e instantes final e inicial (AFS).

\begin{tabular}{|c|c|c|c|c|c|c|c|c|c|c|}
\hline \multirow{2}{*}{ Per. } & \multirow{2}{*}{ Gênero } & \multirow{2}{*}{$\mathbf{N}$} & \multicolumn{4}{|c|}{ Instante inicial } & \multicolumn{4}{|c|}{ Instante final } \\
\hline & & & Média & E.P. & $L_{i}$ & $\mathbf{L}_{\mathrm{s}}$ & Média & E.P. & $L_{i}$ & $\mathbf{L}_{s}$ \\
\hline \multirow{2}{*}{ If/li } & $\mathrm{M}$ & 11 & 45,19 & 0,69 & 43,63 & 46,75 & 46,19 & 0,79 & 44,40 & 47,98 \\
\hline & $\mathrm{F}$ & 11 & 43,65 & 0,48 & 42,56 & 44,74 & 45,11 & 0,50 & 43,99 & 46,25 \\
\hline \multirow{2}{*}{ IIf/IIi } & $\mathrm{M}$ & 7 & 46,53 & 1,12 & 43,65 & 49,41 & 47,67 & 0,87 & 45,43 & 49,91 \\
\hline & $\mathrm{F}$ & 14 & 44,98 & 0,42 & 44,06 & 45,90 & 45,88 & 0,51 & 44,77 & 46,99 \\
\hline \multirow{2}{*}{ IIIf/IIIi } & $M$ & 8 & 45,50 & 1,07 & 42,88 & 48,12 & 45,81 & 1,42 & 42,33 & 49,29 \\
\hline & $\mathrm{F}$ & 10 & 45,15 & 0,78 & 43,35 & 46,95 & 46,76 & 0,79 & 45,01 & 48,51 \\
\hline \multirow{2}{*}{ IVf/IVi } & $\mathrm{M}$ & 7 & 46,98 & 1,30 & 46,64 & 50,32 & 47,83 & 1,31 & 44,46 & 51,20 \\
\hline & $\mathrm{F}$ & 11 & 44,91 & 0,92 & 42,33 & 46,49 & 45,07 & 0,77 & 43,33 & 46,81 \\
\hline \multirow{3}{*}{ (If/IVf) / (li -IVi) } & $\mathrm{M}$ & 33 & 45,93 & 0,49 & 44,93 & 46,93 & 46,76 & 0,54 & 45,66 & 47,86 \\
\hline & $\mathrm{F}$ & 46 & 44,56 & 0,33 & 43,90 & 45,22 & 45,59 & 0,32 & 45,05 & 46,33 \\
\hline & $\mathrm{M}+\mathrm{F}$ & 79 & 45,13 & 0,29 & 44,55 & 45,71 & 46,14 & 0,30 & 45,53 & 46,14 \\
\hline
\end{tabular}

Tabela 5 - Médias, erro-padrão e limites do intervalo segundo período, gênero e instantes final e inicial (AFI).

\begin{tabular}{|c|c|c|c|c|c|c|c|c|c|c|}
\hline \multirow{2}{*}{ Per. } & \multirow{2}{*}{ Gênero } & \multirow{2}{*}{$\mathbf{N}$} & \multicolumn{4}{|c|}{ Instante inicial } & \multicolumn{4}{|c|}{ Instante final } \\
\hline & & & Média & E.P. & $\mathbf{L}_{\mathbf{i}}$ & $\mathbf{L}_{\mathrm{s}}$ & Média & E.P. & $\mathbf{L}_{\mathbf{i}}$ & $L_{s}$ \\
\hline \multirow{2}{*}{ If/li } & $M$ & 11 & 61,72 & 1,62 & 58,06 & 65,38 & 62,50 & 1,72 & 58,61 & 66,39 \\
\hline & $\mathrm{F}$ & 11 & 59,38 & 1,27 & 56,81 & 62,55 & 60,82 & 1,15 & 58,22 & 63,42 \\
\hline \multirow{2}{*}{ IIf/lli } & $M$ & 7 & 63,47 & 1,56 & 59,46 & 67,48 & 64,48 & 1,68 & 60,14 & 68,78 \\
\hline & $\mathrm{F}$ & 14 & 62,73 & 1,28 & 59,94 & 65,52 & 63,53 & 1,18 & 60,96 & 66,10 \\
\hline \multirow{2}{*}{ IIIf/IIII } & M & 8 & 61,00 & 1,31 & 57,79 & 64,21 & 62,45 & 0,87 & 60,32 & 64,58 \\
\hline & $\mathrm{F}$ & 10 & 61,91 & 1,02 & 59,55 & 64,27 & 63,34 & 1,01 & 61,01 & 65,87 \\
\hline \multirow{2}{*}{ IVf/IVi } & $M$ & 7 & 65,66 & 1,53 & 61,73 & 69,59 & 65,96 & 1,60 & 61,85 & 70,07 \\
\hline & $\mathrm{F}$ & 11 & 62,73 & 0,82 & 60,87 & 64,57 & 64,29 & 0,91 & 62,23 & 66,35 \\
\hline \multirow{3}{*}{ (If-IVf) / (li-IVi) } & M & 33 & 62,75 & 0,81 & 61,10 & 64,40 & 63,64 & 0,79 & 62,03 & 65,25 \\
\hline & $\mathrm{F}$ & 46 & 61,82 & 0,59 & 60,63 & 63,01 & 63,02 & 0,56 & 61,89 & 64,15 \\
\hline & $\mathrm{M}+\mathrm{F}$ & 79 & 62,21 & 0,48 & 61,25 & 63,17 & 63,28 & 0,46 & 62,36 & 64,20 \\
\hline
\end{tabular}

\begin{tabular}{|c|c|c|c|c|c|c|c|c|c|c|}
\hline \multirow{2}{*}{ Per. } & \multirow{2}{*}{ Gênero } & \multirow{2}{*}{$\mathbf{N}$} & \multicolumn{4}{|c|}{ Instante inicial } & \multicolumn{4}{|c|}{ Instante final } \\
\hline & & & Média & E.P. & $\mathrm{L}_{\mathrm{i}}$ & $\mathrm{L}_{\mathrm{s}}$ & Média & E.P. & $\mathrm{L}_{\mathrm{i}}$ & $\mathrm{L}_{\mathrm{s}}$ \\
\hline \multirow{2}{*}{ If/li } & $\mathrm{M}$ & 11 & 106,91 & 1,94 & 102,52 & 111,30 & 108,79 & 2,05 & 104,16 & 113,42 \\
\hline & $\mathrm{F}$ & 11 & 103,33 & 1,38 & 100,21 & 106,45 & 105,94 & 1,37 & 102,84 & 109,04 \\
\hline \multirow{2}{*}{ IIf/IIi } & $M$ & 7 & 110,00 & 2,17 & 104,42 & 115,58 & 112,16 & 2,09 & 106,79 & 117,53 \\
\hline & $\mathrm{F}$ & 14 & 107,71 & 1,39 & 104,68 & 110,74 & 109,39 & 1,30 & 106,56 & 112,22 \\
\hline \multirow{2}{*}{ IIIf/IIIi } & M & 8 & 106,50 & 1,74 & 102,24 & 110,76 & 108,21 & 1,63 & 104,22 & 112,20 \\
\hline & $\mathrm{F}$ & 10 & 107,06 & 1,51 & 103,57 & 110,55 & 110,10 & 1,43 & 106,80 & 113,40 \\
\hline \multirow{2}{*}{ IVf/IVi } & M & 7 & 112,64 & 2,13 & 107,16 & 118,12 & 113,79 & 1,91 & 108,88 & 118,70 \\
\hline & $\mathrm{F}$ & 11 & 107,13 & 1,19 & 104,44 & 109,82 & 109,36 & 1,20 & 106,65 & 112,07 \\
\hline \multirow{3}{*}{ (If/IVf) / (li- IVi) } & $\mathrm{M}$ & 33 & 108,68 & 1,05 & 106,54 & 110,82 & 110,42 & 1,03 & 108,32 & 112,53 \\
\hline & $\mathrm{F}$ & 46 & 106,38 & 0,72 & 104,93 & 107,83 & 108,71 & 0,68 & 107,34 & 110,08 \\
\hline & $\mathrm{M}+\mathrm{F}$ & 79 & 107,34 & 0,61 & 106,13 & 108,55 & 109,43 & 0,59 & 108,28 & 110,60 \\
\hline
\end{tabular}


também foram estatisticamente iguais entre si de acordo com as tabelas 4, 5 e 6, com exceção da média da dimensão AFS, no instante inicial, do gênero masculino, no período IVf/IVi. Essa média foi estatisticamente maior do que a do gênero feminino, nos períodos If/Ii, IIf/IIi, IVf/IVi e no período reunião das idades.

A tabela 7 nos aponta que, no instante inicial a medida AFS explicou a variação ocorrida na medida AFT em todas as situações de idade e gênero, com exceção do gênero feminino nos períodos Ii e Ili.

Da mesma maneira, no instante inicial, a medida AFI explicou significativamente a variação ocorrida na medida AFT em ambos os gêneros e em cada idade, em todas as situações.
Mas, esta mesma medida AFI, contribuiu mais do que a medida AFS, para a composição da AFT, no período Ii em ambos os gêneros, no período IIi no gênero feminino, no conjunto das idades (Ii a IVi) em ambos os gêneros e na reunião dos gêneros.

Examinando-se a tabela 8 observamos que no instante final, a AFS explicou a variação ocorrida na medida AFT, em todas as situações de idade e gênero, com exceção das que contemplam o gênero feminino no período IIf e o gênero masculino no periodo IVf.

A medida AFI explicou significativamente a variação ocorrida na medida AFT, em todas as situações, com exceção da relativa ao gênero masculino no período IIIf. Mas, a medida AFI contribuiu mais

\begin{tabular}{|c|c|c|c|c|c|c|c|c|c|c|}
\hline \multirow{2}{*}{ Período } & \multirow{2}{*}{ Gênero } & \multirow{2}{*}{$\mathbf{N}$} & \multicolumn{3}{|c|}{ AFS } & \multicolumn{3}{|c|}{ AFI } & \multirow{2}{*}{$\begin{array}{c}\text { AFS } x \\
Z_{0}\end{array}$} & \multirow{2}{*}{$\begin{array}{c}\text { AFI } \\
\mathbf{p}\end{array}$} \\
\hline & & & $\mathrm{CD}(\%)$ & $z_{0}$ & $\mathbf{p}$ & $\mathrm{CD}(\%)$ & $\mathrm{Z}_{0}$ & $\mathbf{p}$ & & \\
\hline \multirow{2}{*}{ li } & $\mathrm{M}$ & 11 & 35,8 & $2,41^{*}$ & 0,01 & 88,4 & $9,21^{*}$ & 0,00 & $2,73^{*}$ & 0,01 \\
\hline & $F$ & 11 & 15,2 & $1,41 \mathrm{n}$ & 0,16 & 82,8 & $8,64^{*}$ & 0,00 & -- & -- \\
\hline \multirow{2}{*}{ lii } & $M$ & 7 & 52,1 & $2,76^{*}$ & 0,01 & 75,2 & $4,61^{*}$ & 0,00 & $0,91 n$ & 0,36 \\
\hline & $F$ & 14 & 15,9 & $1.62 n$ & 0,11 & 90,9 & $11,80^{*}$ & 0,00 & -- & -- \\
\hline \multirow{2}{*}{ Illi } & $M$ & 8 & 43,1 & $2,46^{*}$ & 0,01 & 62,5 & $3,65^{*}$ & 0,00 & $0,73 n$ & 0,46 \\
\hline & $\mathrm{F}$ & 10 & 61,8 & $4,01^{*}$ & 0,00 & 77,3 & $5,86^{*}$ & 0,00 & $0,75 n$ & 0,45 \\
\hline \multirow{2}{*}{ IVi } & $\mathrm{M}$ & 7 & 49,2 & $3,30^{*}$ & 0,00 & 63,2 & $3,90^{*}$ & 0,00 & $0,53 n$ & 0,60 \\
\hline & $\mathrm{F}$ & 11 & 52,5 & $3,50^{*}$ & 0,00 & 41,4 & $2,79^{*}$ & 0,01 & $0,52 n$ & 0,60 \\
\hline \multirow{3}{*}{ li a IVi } & $M$ & 33 & 45,2 & $5,26^{*}$ & 0,00 & 79,4 & $11,34^{*}$ & 0,00 & $2,94^{*}$ & 0,01 \\
\hline & $\mathrm{F}$ & 46 & 34,2 & $4,89^{*}$ & 0,00 & 79,7 & $13,28^{*}$ & 0,00 & $4,59^{*}$ & 0,00 \\
\hline & $M+F$ & 79 & 42,3 & $7,69^{*}$ & 0,00 & 79,5 & $17,67^{*}$ & 0,00 & $4,94^{*}$ & 0,00 \\
\hline
\end{tabular}

* = valor significante; $\mathrm{n}$ = valor não significante.

Tabela 8 - Coeficiente de determinação entre AFT e AFS ou AFI e valores $Z_{0}$ e p, segundo período e gênero no instante final.

\begin{tabular}{|c|c|c|c|c|c|c|c|c|c|c|}
\hline \multirow{2}{*}{ Período } & \multirow{2}{*}{ Gênero } & \multirow{2}{*}{$\mathbf{N}$} & \multicolumn{3}{|c|}{ AFS } & \multicolumn{3}{|c|}{ AFI } & \multirow{2}{*}{$\begin{array}{c}\text { AFS } x \\
Z_{0}\end{array}$} & \multirow{2}{*}{$\begin{array}{c}\text { AFI } \\
\mathbf{p}\end{array}$} \\
\hline & & & $\mathrm{CD}(\%)$ & $z_{0}$ & p & CD(\%) & $Z_{0}$ & p & & \\
\hline \multirow{2}{*}{ If } & $\mathrm{M}$ & 11 & 29,4 & $2,15^{*}$ & 0,03 & 84,9 & $7,86^{*}$ & 0,00 & $2,81^{*}$ & 0,01 \\
\hline & $\mathrm{F}$ & 11 & 35,1 & $2,44^{*}$ & 0,12 & 87,5 & $8,75^{*}$ & 0,00 & $2,70^{*}$ & 0,01 \\
\hline \multirow{2}{*}{ Ilf } & $M$ & 7 & 39,2 & $2,12^{*}$ & 0,03 & 83,9 & $6,08^{*}$ & 0,00 & $1,78 \mathrm{n}$ & 0,07 \\
\hline & $\mathrm{F}$ & 14 & 17,1 & $1.71 \mathrm{n}$ & 0,09 & 84,9 & $8,84^{*}$ & 0,00 & -- & -- \\
\hline \multirow{2}{*}{ IIIf } & $M$ & 8 & 73,4 & $4,70^{*}$ & 0,00 & 26,4 & $1,69 n$ & 0,09 & -- & -- \\
\hline & $\mathrm{F}$ & 10 & 53,9 & $3,39^{*}$ & 0,00 & 71,4 & $4,99^{*}$ & 0,00 & $0,81 \mathrm{n}$ & 0,42 \\
\hline \multirow{2}{*}{ IVf } & $M$ & 7 & 31,0 & $1,77 n$ & 0,08 & 54,0 & $2,87^{*}$ & 0,00 & -- & -- \\
\hline & $\mathrm{F}$ & 11 & 42,0 & $2,82^{*}$ & 0,01 & 58,9 & $3,98^{*}$ & 0,00 & $0,80 \mathrm{n}$ & 0,42 \\
\hline \multirow{3}{*}{ If a IVf } & $M$ & 33 & 41,1 & $4,78^{*}$ & 0,00 & 72,9 & $9,47^{*}$ & 0,00 & $2,66^{*}$ & 0,01 \\
\hline & $F$ & 46 & 33,5 & $4,85^{*}$ & 0,00 & 78,2 & $12,82^{*}$ & 0,00 & $4,49^{*}$ & 0,00 \\
\hline & $\mathrm{M}+\mathrm{F}$ & 79 & 39,0 & $7,09^{*}$ & 0,00 & 75,0 & $15,31^{*}$ & 0,00 & $4,68^{*}$ & 0,00 \\
\hline
\end{tabular}

* $=$ valor significante; $\mathrm{n}$ = valor não significante. 
do que a medida AFS, para a composição da AFT, no período If em ambos os gêneros, no período IIf no gênero feminino, no conjunto das idades (If a IVf) em ambos os gêneros e na reunião dos gêneros.

Portanto, observando-se as tabelas 7 e 8 , podemos notar que a AFI teve tendência a influenciar mais na dimensão AFT do que a AFS, que, portanto, ficou um pouco mais estável, demonstrando que a face inferior se modifica mais do que a face média, durante o crescimento.

Com relação às proporções faciais anteriores, podemos observar na tabela 9 que elas se mantiveram constantes em todas as situações, mantendo percentagem aproximada de $42 \%$ para a dimensão AFS e, portanto, $58 \%$ para a dimensão AFI.

\section{DISCUSSÃO}

A proporção facial equilibrada em todas as idades mostrada na tabela 9 , está em consonância com a grande maioria dos autores pesquisa$\operatorname{dos}^{03,04,07,15,22,24,30,33,33,38}$, que apesar de encontrarem proporções faciais levemente diferentes, elas se mantêm relativamente constantes e se estabelecem precocemente.

Com relação ao dimorfismo sexual, a dimensão AFT, apresentou-se levemente maior nos indivíduos, do gênero masculino, com exceção das médias encontradas no período IIIf/IIIi, quando os valores nos indivíduos do gênero feminino foram iguais ou maiores do que os do gênero masculino, demonstrando um possível surto máximo de crescimento, neste período, no gênero feminino, apesar de estas dimensões não terem sido estatisticamente significantes. A dimensão AFT maior nos indivíduos do gênero masculino está em sintonia com as observações feitas pelos autores pesquisados ${ }^{05,06,07,17,20,22,23,28,38}$.

Notamos que as dimensões AFSf, AFIf, AFTf, AFPf, AFAf e IAFf tiveram tendência a valores maiores no conjunto das idades, somente no gênero feminino, confirmando um possível surto de crescimento maior destes indivíduos, até a idade de 11 anos, enquanto que, os do gênero masculino, teriam este crescimento, após esta idade.

Nas tabelas 7 e 8 podemos observar que a AFI teve tendência a influenciar mais na dimensão AFT do que a AFS, que, portanto, ficou um pouco mais estável. Esta mesma observação foi constatada pelos autores pesquisados ${ }^{13,19,14,31,26,29,25,10,30,18,21}$.

O IAF obtido neste trabalho permaneceu constante ou teve uma tendência a aumentar com a idade, portanto, estando em concordância com a tendência normal de crescimento, quando a mandíbula tem uma rotação no sentido anti-horário, aumentando, por conseguinte o IAF ${ }^{01,12}$.

A tabela 3 evidenciou que o IAF médio foi de 0,66 ao passo que $\mathrm{Schudy}^{30}$ e Horn ${ }^{16}$ encontraram valores médios de 0,68 e 0,70 respectivamente.

\begin{tabular}{|c|c|c|c|c|c|c|c|c|c|}
\hline \multicolumn{10}{|c|}{$\begin{array}{c}\text { Tabela } 9 \text { - Médias, erros-padrão e limites de confiança para o percentual da AFS } \\
\text { relativamente à AFT nos instantes inicial e final. }\end{array}$} \\
\hline \multirow{2}{*}{ Per. } & \multirow{2}{*}{ Gênero } & \multicolumn{4}{|c|}{ Instante inicial } & \multicolumn{4}{|c|}{ Instante final } \\
\hline & & Média & E.P. & Li & Ls & Média & E.P. & Li & Ls \\
\hline \multirow{2}{*}{ If $/ \mathrm{li}$} & M & 42,34 & 0,65 & 40,91 & 43,76 & 42,54 & 0,75 & 40,87 & 44,21 \\
\hline & $\mathrm{F}$ & 42,29 & 0,59 & 40,12 & 43,60 & 42,63 & 0,48 & 41,56 & 43,90 \\
\hline \multirow{2}{*}{ IIf/lii } & M & 42,31 & 0,72 & 40,55 & 44,07 & 42,54 & 0,69 & 40,90 & 44,18 \\
\hline & $\mathrm{F}$ & 41,83 & 0,52 & 40,71 & 42,95 & 41,99 & 0,52 & 40,87 & 43,11 \\
\hline \multirow{2}{*}{ IIIf/IIli } & $M$ & 42,73 & 0,78 & 40,88 & 44,57 & 42,28 & 0,84 & 40,64 & 44,27 \\
\hline & $\mathrm{F}$ & 42,17 & 0,45 & 41,15 & 43,19 & 42,47 & 0,49 & 41,36 & 43,58 \\
\hline \multirow{2}{*}{ IVf/lvi } & M & 41,71 & 0,85 & 40,23 & 43,79 & 42,04 & 1,00 & 39,59 & 44,49 \\
\hline & $\mathrm{F}$ & 41,44 & 0,61 & 40,08 & 42,80 & 41,21 & 0,54 & 40,01 & 42,41 \\
\hline \multirow{3}{*}{ (If/IVf) / (li- IVi) } & M & 42,29 & 0,36 & 41,55 & 43,02 & 42,37 & 0,39 & 41,57 & 43,17 \\
\hline & $\mathrm{F}$ & 41,92 & 0,27 & 41,38 & 42,46 & 42,06 & 0,26 & 41,49 & 42,53 \\
\hline & $\mathrm{M}+\mathrm{F}$ & 42,08 & 0,22 & 41,64 & 42,52 & 42,19 & 0,22 & 41,75 & 42,63 \\
\hline
\end{tabular}


Portanto, como este índice médio do presente trabalho se apresentou mais baixo, isso nos leva a pensar que os indivíduos da amostra estudada têm um padrão um pouco mais vertical, mas mantendo-se dentro dos limites dos casos considerados não cirúrgicos ${ }^{16}$.

Em função da relação que existe entre AFA e AFP, vários autores ${ }^{12,16,35}$, propõem a utilização do Índice da Altura Facial no diagnóstico e plano de tratamento, pois ele representa, de uma maneira bastante clara, a rotação mandibular na fase de crescimento ou o comportamento desta rotação, com o tratamento ortodôntico. $\mathrm{O}$ aumento da razão AFP/AFA, isto é, do IAF, nos casos tratados, deve ser considerado um fator principal na manutenção da correção da má oclusão Classe II $^{35}$. O valor do IAF pode ajudar o clínico a tomar decisão antes do tratamento, para avaliar o crescimento rotacional da mandíbula. Através de cefalogramas seriados feitos antes do tratamento, o IAF pode fornecer uma indicação da tendência da rotação mandibular, que poderá ser para cima e para frente, se o IAF aumentar e para baixo e para trás, se o IAF diminuir.

\section{CONCLUSÕES}

De acordo com os resultados desta pesquisa, em que foram estudados indivíduos portadores de má oclusão Classe I de Angle, com padrões verticais variados, consideramos lícito concluir que:

1) $O$ crescimento das dimensões faciais anterior e posterior analisadas, manteve-se constante e equilibrado, em todos os períodos estudados.

2) O Índice da Altura Facial médio foi de 0,66 e não diminuiu com a idade, se mantendo constante ou aumentando.

3) Não houve dimorfismo sexual, tanto com relação às proporções faciais, como também, com relação ao Índice da Altura Facial.

4) As proporções faciais anteriores se mantiveram em torno de $42 \%$ para dimensão altura facial superior e $58 \%$ para a dimensão altura facial inferior.

5) A dimensão altura facial inferior, contribuiu de forma mais significativa nas alterações observadas na altura facial total.

\title{
Cephalometric study of the anterior and posterior facial heights in brazilian children, presenting Angle Class I malocclusion, in the mixed dentition
}

\begin{abstract}
The objective of this study was to evaluate the anterior and posterior facial heights in 79 non orthodontic brazilian children, 46 females and 33 males, from 8 to 11 years old, presenting Angle Class I malocclusion, in the mixed dentition. Two lateral cephalometric radiographs were taken, with a time interval ranging from 8 to 16 months between radiographs in order to compare the cephalometric data. The anterior facial proportions were studied, based in following dimensions: N-Me (AFT), N-ENA (AFS) e ENA-Me (AFI). The Facial Height Index (IAF) was also evaluated taking in account the relationship between the Ar-Go (AFP) and the distance between the palatal plane and the menton (AFA). The results showed that growth tendencies were balanced; the Lower Facial Height dimension contributed more significantly to the Total Facial Height changes; the anterior facial proportions were around 42\% for the Upper Facial Height and 58\% for the Lower Facial height; the Facial Height Index was stable, around 0,66 or had a tendency to increase, according to the age; there were no sexual dimorphism in Anterior Facial Proportion and Facial Height Index.
\end{abstract}

Key words: Cephalometric. Growth. Skeletal pattern. 


\section{REFERÊNCIAS}

1. BISHARA, S. E. et al. Changes in facial dimensions and relationships between the ages of 5 and 25 years. Am J Orthod, St. Louis, v. 85, p. 238-252, 1984.

2. BISHARA, S. E.; JAKOBSEN, J. R. Longitudinal changes in three normal facial types. Am J Orthod, St. Louis, v. 88, p. 466-502, 1985.

3. BRODIE, A. G. On the growth pattern of the human head from the third month to the eight years. Am J Anat, Philadelphia, v. 68, p. 209-262, 1941.

4. BRODIE, A. G. On the growth of the jaws and the eruption of the teeth. J Dept Orthod, [S. I.], v. 12, p.109-123, 1942.

5. CANNON, J. Craniofacial height and depth increments in normal children. Angle Orthod, Appleton, v. 40, p. 202-218, 1970.

6. COBEN, S. E. The integration of facial skeletal variants. Am J Orthod, St. Louis, v. 41, p. 407-434, 1955

7. DOMITTI, S. S.; DARUGE, E.; CRUZ, V. F. Variability of the nasion-subnasal, subnasal-gnation and bizygomatic distances of individuals of $6,7,11$ and 15 years of age and their importance in the determination of the vertical dimension. Aust Dent J Sydney, v. 21, p. 269-271, 1976.

8. DOWNS, W. B. Variations in facial relationships: their significance in treatment and prognosis. Am J Orthod, St. Louis, v. 34, p. 812-840, 1948.

9. DOWNS, W.B. Analysis of the dentofacial profile. Angle Orthod, Appleton, v. 26, p.191-212, 1956.

10. FIELDS, H. W. et al. Facial pattern differences in long-faced children and adult. Am J Orthod, St. Louis, v. 85, p. 217-223, 1984.

11. GEBECK, R. T.; MERRIFIELD, L. L. Analysis: concepts and values. Part I. J Charles H Tweed Int Found, California, v.17, p.1948, 1989.

12. GEBECK, R.T.; MERRIFIELD, L. L. Analysis: concepts and values. Part II. J Charles H Tweed Int Found, California, v.17, p. 49-64, 1989.

13. GOLDSMAN, S. The variations in skeletal and denture patterns in excellent adult facial types. Angle Orthod, Appleton, v. 29, p. 63-92, 1959.

14. HAPAK, F. M. Cephalometric appraisal of the open-bite case. Angle Orthod, Appleton, v. 34, p. 65-72, 1964.

15. HERZBERG, F.; HOLIC, R. An antropologic study of face height. Am J Orthod Oral Surg, St. Louis, v. 29, p. 90-100, 1943.

16. HORN, A. J. Facial height index. Am J Orthod, St. Louis, v.102, p.180-186, 1992.

17. HOROWITZ, S. L.; THOMPSON JR., R. H. Variations of the craniofacial skeletal in postadolescent males and females. Angle Orthod, Appleton, v. 34, p. 97-102, 1964.

18. ISAACSON, J. R. et al. Extreme variation in vertical facial growth and associated variation in skeletal and dental relations. Angle Orthod, Appleton, v. 41, p. 219-229, 1971.

19. JOHNSON, E. L. The Frankfort - Mandibular plane Angle and the facial pattern. Am J Orthod, St. Louis, v. 36, p. 516-533, 1950.
20. JONES, B. H.; MEREDITH, V. H. Vertical change in osseous and odontic portions of human face height between the ages of 5 and 15 years. Am J Orthod, St. Louis, v. 52, p. 902-921, 1966.

21. KERR, W. J. S.; LAN FORD, B. S. The variability of some craniofacial dimensions. Angle Orthod, Appleton, v. 61, p. 205-210, 1991.

22. LOCKS, A. Análise das proporções verticais anteriores da face de indivíduos brasileiros, portadores de oclusão excelente e perfil agradável. 1981. 69 f. Dissertação (Mestrado) Universidade Federal, Rio de Janeiro, 1981.

23. NAHOUM, H. I. Vertical proportions and the palatal plane in anterior open-bite. Am J Orthod, St. Louis, v. 59, p. 273-282, 1971.

24. NANDA, S. K. Patterns of vertical growth in the face. Am J Orthod Dentofacial Orthop, St. Louis, v. 93, p.103-116, 1988.

25. OPDEBEECK, H. et al. Comparative study between the SFS and LFS relation as a possible morphogenic mechanism. Am J Orthod, St. Louis, v. 74, p. 510-521, 1978

26. RICHARDSON, A. Skeletal factors in anterior open bite and deep bite. Am J Orthod, St. Louis, v. 56, p.114-127, 1969.

27. SASSOUNI, V.; NANDA, S. Analysis of dentofacial vertical proportions. Am J Orthod, St. Louis, v. 50, p. 801-823, 1964.

28. SCHEIDEMAN, G. B. et al. Cephalometric analysis of dentofacial normals. Am J Orthod, St. Louis, v. 78, p. 404-420, 1980.

29. SCHENDEL, S. A. et al. The long face syndrome: vertical maxillary excess. Am J Orthod, St. Louis, v. 70, p. 398-408, 1976.

30. SCHUDY, F. F. Vertical growth versus antero-posterior growth as related to function and treatment. Angle Orthod, Appleton, v. 34, p. 75-93, 1964.

31. SUBTELNY, J. D.; SAKUDA, M. Open bite: diagnosis and treatment. Am J Orthod, St. Louis, v. 50, p. 337-358, 1964.

32. THOMPSON, J. R.; BRODIE, A. G. Factors in the position of the mandible. Am J Orthod, St. Louis, v. 29, p. 925-941, 1942.

33. TIRK, T. M. A study of the growth of the head by planimetric method. Angle Orthod, Appleton, v. 28, p.76-94, 1948.

34. TWEED, C. H. Indications for the extraction of teeth in orthodontic procedure. Am J Orthod Oral Surgery, St. Louis, v. 30, p. 405-428, 1944.

35. VADEN, J. L.; HARRIS, E. F.; SINCLAIR, P. M. Clinical ramifications of posterior and anterior facial height changes between treated and untreated class II samples. Am J Orthod Dentofacial Orthop, St. Louis, v. 105, p. 438-443, 1994.

36. WOLK, K.; SANDERS, B. Pediatric oral and maxilofacial surgery. St. Louis: C. V. Mosby, 1979. cap.13, p. ?

37. WYLIE, W. L. Statistical analysis of the constancy of certain facial dimensions. J Dent Res, Chicago, v. 22, p. 200-201, 1944.

38. WYLIE, W. L.; JOHNSON, E. L. Rapid evaluation of facial dysplasia in the vertical plane. Angle Orthod, Appleton, v. 22, p.165$182,1952$.

\author{
Endereço para correspondência \\ Arno Locks \\ Rua Presidente Coutinho, 311, bloco A, sala 1101 \\ CEP: 88015-230 \\ E-mail: arnoorto@terra.com.br
}

